001 APPROPRIATE USE OF DOWN-STREAM INVASIVE CORONARY ANGIOGRAPHY (ICA) FOLLOWING COMPUTED TOMOGRAPHIC CORONARY ANGIOGRAPHY (CTCA) IS STILL A WORK IN PROGRESS

${ }^{1}$ Mohammed 0 Abubakr, ${ }^{2}$ Carl A Roobottom, ${ }^{1}$ Gareth J Morgan-Hughes. ${ }^{1}$ Cardiology Department, Plymouth Hospitals NHS Trust, Plymouth, UK; ${ }^{2}$ Radiology Department, Plymouth Hospitals NHS Trust, Plymouth, UK

\subsection{6/heartjnl-2016-309680.1}

Introduction There is little difference between CTCA and ICA in the ability to predict ICA fractional flow reverse (FFR) positive stenosis and ICA should follow CTCA to facilitate revascularisation not to 'check' CTCA results. This audit determined the proportion of patients having no treatment change (NTC) when ICA followed CTCA in Plymouth Hospitals NHS Trust (PHNT).

Methods A retrospective single site audit identified 292 patients who had both diagnostic quality CTCA and subsequent ICA for the same patient episode between 2013 and 2014. Significant coronary artery disease (CAD) was defined as $>50 \%$ stenosis $(\geq$ moderate) and ICA was used as the reference standard (deferring to FFR if available). Patients were classified according to whether revascularisation followed ICA, or NTC resulted in which case we identified a main factor why.

Results There was 89\% agreement between CTCA and invasive testing. 27/292 (9.25\%) patients had CTCA considered false positive and 5/292 (1.71\%) false negative (FN) after ICA +/- FFR (33/292 FFR, 11.30\%). Most patients were revascularised 168/ 292 (57.53\%, PCI 119/292, (40.75\%), CABG 49/292 (16.78\%)) with the CTCA report suggesting revascularisation in $157 / 292$ (53.77\%). NTC group consisted of 124/292 (42.47\%) patients. $53 / 292(18.15 \%)$ treated with medical therapy, 64/292 (21.92\%) had atypical symptoms, 5/292 (1.71\%) had complex comorbidities and 2/292 (0.68\%) had other reasons.

Conclusion CTCA accuracy was high. However $42 \%$ of patients undergoing ICA following CTCA have NTC resulting. A positive CTCA result should therefore prompt consideration of clinical consultation, functional imaging or ICA rather than direct ICA.

\section{CARDIAC CT: PREDICTOR OF REVASCULARISATION}

${ }^{1} \mathrm{~A}$ Beattie, ${ }^{2} \mathrm{M}$ Albarri, ${ }^{1} \mathrm{R}$ McStay, ${ }^{1}$ Lisa Andrews. ${ }^{1}$ The Newcastle-Upon-Tyne Hospitals Trust, Newcastle, UK; ${ }^{2}$ Newcastle University, Newcastle, UK

10.1136/heartjnl-2016-309680.2

Introduction 2010 NICE guidelines recommend cardiac CT in patients with stable chest pain and likelihood of coronary artery disease of $10-29 \%$. We determined use of correlative imaging to assess for coronary artery disease and subsequent revascularisation.

Methods All patients who underwent cardiac CT for chest pain assessment between September 2011 and March 2013 were identified. Those with evidence of previous revascularisation were excluded. Presence and severity of reported coronary disease was documented. Correlative cardiac imaging and revascularisation were identified from electronic patient records.

Results Of 552 chest pain patients referred for cardiac CT, 293 had calcium scoring only (CTCS), 211 had CTCS and CT Coronary Angiogram (CTCA) and 48 had CTCA only. 49 patients underwent subsequent invasive angiography (IA) (8.9\% patients). 14 of those were revascularised $(2.5 \%$ all patients), all with abnormal CTs: 2 had CABG and 12 percutaneous coronary intervention. Of the 35 patients not revascularised, 5 had normal CTCA and IA, 24 had positive CT and negative IA, 6 had both positive. Of the patients undergoing IA, 4 of 28 (14.3\%) with a calcium score $<400$ were revascularised compared with 9 of the $17(52.3 \%)$ with a calcium score $>400$.

Conclusions $2.5 \%$ of patients referred for cardiac CT to investigate chest pain without prior interventional treatment undergo revascularisation. Revascularisation rates in patients with a positive CTCA and a calcium score of $<400$ were only $14.3 \%$ compared with $52.3 \%$ in those with scores of $>400$. This suggests a score $<400$ may help reduce unnecessary IA.

\section{DETECTING HYPERTENSIVE HEART DISEASE: THE ADDITIVE VALUE OF CARDIOVASCULAR MAGNETIC RESONANCE IMAGING}

${ }^{1}$ Max Charalambos, ${ }^{2,3}$ Jonathan Rodrigues, ${ }^{4}$ Amy Burchell, ${ }^{2}$ Amardeep Ghosh Dastidar ${ }^{4}$ Laura Ratcliffe, ${ }^{4}$ Emma Hart, ${ }^{2}$ Mark Hamilton, ${ }^{3,4}$ Julian Paton, ${ }^{4}$ Angus Nightingale, ${ }^{2}$ Nathan Manghat. 'Medical School, University of Bristol, UK; ${ }^{2}$ NIHR Bristol Cardiovascular Biomedical Research Unit, Bristol Heart Institute, University Hospitals Bristol NHS Foundation Trust, UK; ${ }^{3}$ School of Physiology, Pharmacology and Neuroscience, Faculty of Biomedical Science, Medical Sciences Building, University of Bristol, UK; ${ }^{4}$ CardioNomics Research Group, Clinical Imaging and Research Centre, Bristol Heart Institute, University Hospitals Bristol NHS Foundation Trust, UK

\subsection{6/heartjnl-2016-309680.3}

Introduction International hypertension guidelines advise screening for hypertensive heart disease (HHD) to aid risk stratification. Cardiac magnetic resonance (CMR) is the current noninvasive gold-standard for assessing ventricular structure/function. We aimed to determine the additive value of CMR in hypertensives over echocardiography.

Methods 85 subjects $(60 \%$ men, office systolic BP: $165 \pm$ $28 \mathrm{mmHg}$, office diastolic BP: $94 \pm 14 \mathrm{mmHg}$ ) from our tertiary hypertension clinic with preceding echo underwent 1.5T CMR. Left ventricular mass and volumes were estimated from shortaxis steady-state free precession cines. $\mathrm{LVH}$ was defined on the basis of echo and CMR normal reference ranges. The presence and pattern of myocardial late gadolinium enhancement (LGE) was documented.

Results Overall, there was no difference in prevalence of $\mathrm{LVH}$ by echo compared to CMR (68\% vs $66 \%, \mathrm{P}=0.746)$. However, there was a discrepancy between echo and CMR in $28 \%$. Relative to CMR gold-standard, echo over-diagnosed LVH in $15 \%$ and missed $\mathrm{LVH}$ in $13 \%$. The diagnostic performance of echo at detecting LVH was as follows: specificity 55\%, sensitivity $80 \%$, positive predictive value $78 \%$, negative predictive value $59 \%$ and overall accuracy $72 \%$. Ischaemic LGE was present in $7 \%$ of subjects and non-ischaemic LGE was present in 9\%.

Conclusion Echocardiography over-diagnosed and under-diagnosed LVH in an important minority of patients. LGE tissue characterisation is unique to CMR and identified ischaemic and non-ischaemic myocardial fibrosis is an important minority of hypertensives. Our findings support an extended role of CMR in hypertension where documenting in the presence/absence of HHD may have clinical management implications. 